\title{
Multi-wavelength Brillouin Fiber Laser with Fiber Bragg Grating Employs Loop Mirror
}

\author{
N.A. Idris ${ }^{1,{ }^{*}}$, N.A.M.A. Hambali ${ }^{1}$, A.Z. Malek ${ }^{1}$, N.H.M. Apandi ${ }^{1}$, M.H.A. Wahid $^{1}$, M.A.M. \\ Azidin $^{1}$, and S.S.M. Isa ${ }^{1}$ \\ ${ }^{1}$ Semiconductor Photonics \& Integrated Lightwave Systems (SPILS), School of Microelectronic \\ Engineering, Universiti Malaysia Perlis, Pauh Putra, 02600 Arau, Perlis, Malaysia.
}

\begin{abstract}
In this paper, the development of multi-wavelength Brillouin fiber laser with fiber Bragg grating utilizing a loop mirror is successfully demonstrated. A multi-wavelength Brillouin fiber laser configuration employs $11 \mathrm{~km}$ length of single mode fiber as the gain medium, while the reflection of fiber Bragg grating is adopted to produce multi-wavelength signals. Based on the experimental results, it shows that the position of the fiber Bragg grating in the Brillouin fiber laser configuration plays an important role in producing the multi-wavelength signals. By using 19.0 $\mathrm{dBm}$ of Brillouin pump power, the highest number of Brillouin Stokes signal and tuning range obtained from this formation is about 23 and $4 \mathrm{~nm}$, respectively. Furthermore, $50 \%$ output coupling ratio demonstrates the best ratio to produce Brillouin Stokes signal which gives a better performance in the Brillouin fiber laser system.
\end{abstract}

\section{Introduction}

Generally, the growth towards the communication is rapidly increasing with the higher demands on the data bandwidth which led to the development of the optical fiber and the exploration on the long haul transmission media [1-6]. The advancement of the optical fiber technology is continued by the invention of fiber lasers where the laser resonator is made up by selective fiber materials [7]. The advantages of fiber laser in mainly due to better reliability lifetime, high power, high stability, low amplitude noise, high output stability, immune to tough environment changes, low threshold power and narrow line width [8]. These advantages have extensive application such as optical sensing [9], optical filtering [10], narrow-bandwidth amplification [11], high-resolution spectroscopy [12], and optical fiber communications.

Brillouin fiber laser (BFL) is a type of fiber laser that utilizes stimulated Brillouin scattering (SBS) nonlinear effect. The SBS is a nonlinear process in optical fiber which involves the interaction between intense pump light and acoustic wave [13]. This process produces a unique result where Brillouin Stokes (BS) signals are generated in backward propagation at a downshifted frequency of $\sim 0.08 \mathrm{~nm}(10 \mathrm{GHz})$ from the injected signal wavelength [13].

\footnotetext{
*Corresponding author: athirah.idris@yahoo.com
} 
The implementation of the fiber Bragg grating (FBG) and loop mirror in the BFL configuration plays an important role in generating the multi-wavelength signal. This FBG is known as the optical fiber core that possess a special disorder refractive index region which it will diffracts the light ray propagation at a particular wavelength [14]. The loop mirror is a reflecting structure that acts as the mirror and help the generation of the BS signals [15]. On the other hand, this configuration has been conducted in the C-band in the range of $1530 \mathrm{~nm}$ to $1565 \mathrm{~nm}$ transmission wavelength. This band promotes the lowest attenuation and compatible system with amplifiers [16].

In this paper, the performance of multi-wavelength Brillouin fiber laser (MWBFL) with FBG utilizing loop mirror is demonstrated. The fiber loop acts as a mirror in such a way that it is able to reflect back most of the input signal. Evidently, the $50 \%$ output coupling ratio shows the best ratio of Brillouin pump power to stoke power which gives the better performance in this configuration. At $50 \%$ output coupling ratio and $19.0 \mathrm{dBm}$ of $\mathrm{BP}$ power, $23 \mathrm{BS}$ signals are produced. In addition, this configuration has been operated in the C-band region in the range of $1530 \mathrm{~nm}$ to $1565 \mathrm{~nm}$ transmission wavelength. This band promotes the lowest attenuation and compatible system with amplifiers [16].

\section{Experimental setup and principle}

The basic configuration of MBFL utilizing loop mirror is shown in Fig. 1. Based on this setup, the external tunable laser source (TLS) is utilized as a Brillouin pump (BP) signal. Besides that, $11 \mathrm{~km}$ length of single mode fiber (SMF) functions as Brillouin gain medium. A reflection of the FBG is the main factor in producing multi-wavelength in FBL configuration. The FBG has central wavelength of $1550 \mathrm{~nm}$ and $3 \mathrm{~dB}$ bandwidth of $1 \mathrm{~nm}$ with $90 \%$ reflectivity. The bidirectional optical coupler possesses the coupling ratio of $20 \%, 30 \%, 50 \%, 70 \%$ and $80 \%$. Erbium-doped fiber amplifier (EDFA) amplifies the BP power in the range of $16 \mathrm{dBm}$ to $20 \mathrm{dBm}$ before going through an optical circulator. The optical circulator avoids the BP signal to be redirected to the source. An optical spectrum analyzer (OSA) monitors the output signals of MBFL.

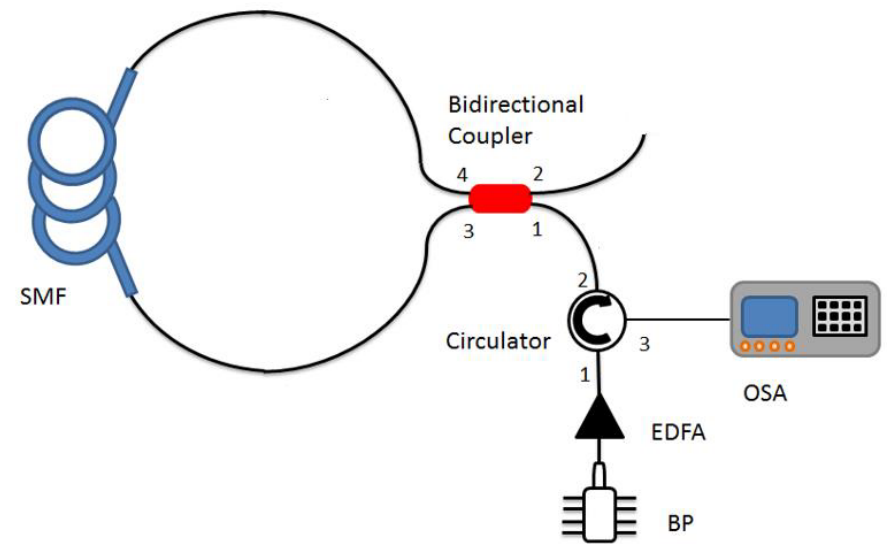

Fig.2. The basic configuration of the MBFL utilizing loop mirror.

The operation is started by the injection of the Brillouin pump (BP) signal via external TLS into the EDFA. The EDFA amplified and produced stronger BP signal in the loop [17]. Next, the signal is travelled through the optical circulator where the signals are conducted from one port to another and also has a function to prevent the signal to propagate in random directions [18]. The BP signal is then undergone the bidirectional optical coupler and propagated into the gain medium of the SMF. After achieving the 
Brillouin threshold in SMF, the Brillouin Stokes (BS) signal is generated with the down shifted of $\sim 0.08 \mathrm{~nm}$ from the initial BP signal wavelength and propagated in backward direction. This signal is the first generation of the BS signal. The second generation of the BS signal is produced when the signal propagated to the FBG reflects back into the loop mirror, thus creating the second generation of BS signal. Finally, the BS signal is directed to optical spectrum analyzer (OSA) through the bidirectional optical coupler and optical circulator. The observation and measurement of the output results is performed using the OSA with resolution bandwidth of $0.02 \mathrm{~nm}$.

\section{Experimental result and discussion}

Producing a narrow linewidth multi-wavelength in the BFL setup of this work requires the position of FBG at different locations. Fig. 2 depicts three different locations of FBG. The FBG is placed in the loop mirror which is located after and before SMF as shown in Fig. 2(a) and Fig. 2(b), respectively. Whereas the FBG placed at the end is illustrated in Fig. 2(c).

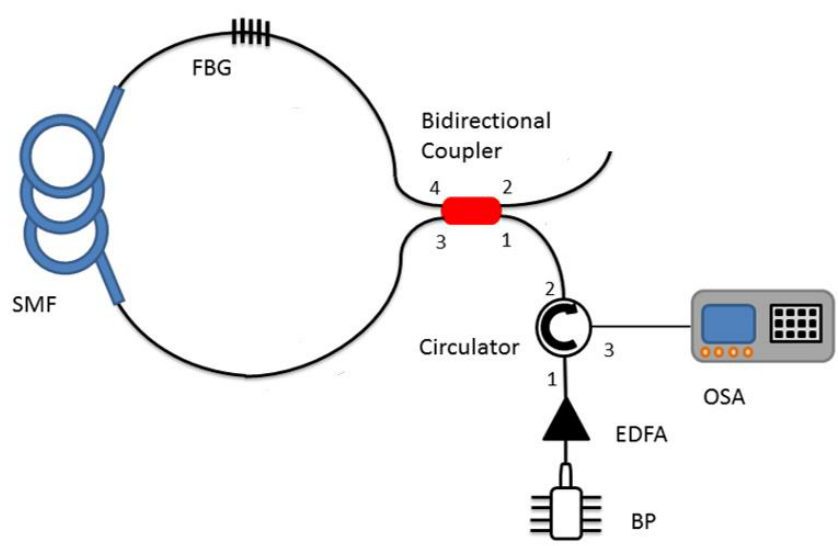

(a)

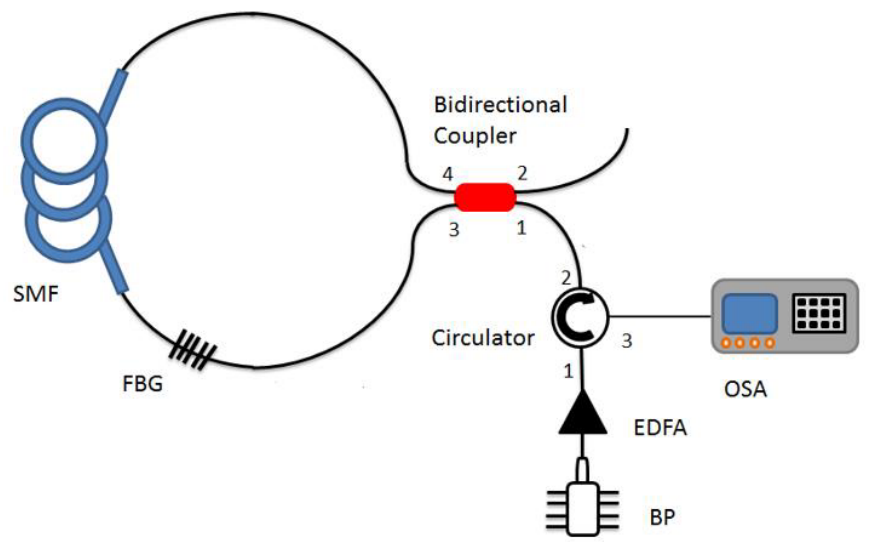

(b) 


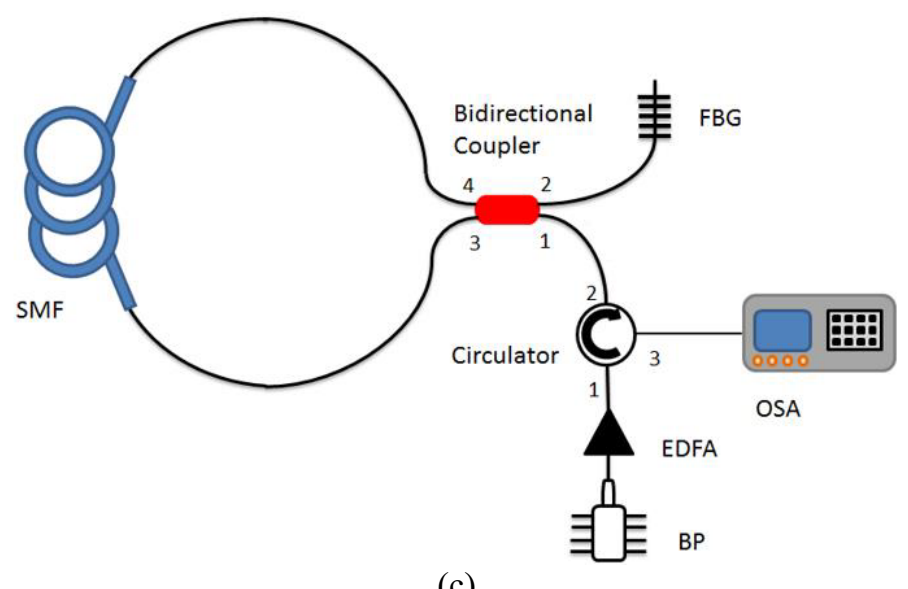

(c)

Fig. 2. The different positions of FBG in the MBFL configuration. (a) The FBG is placed in the loop mirror after the SMF. (b) The FBG is placed in loop mirror before the SMF. (c) The FBG is placed at the end of the configuration.

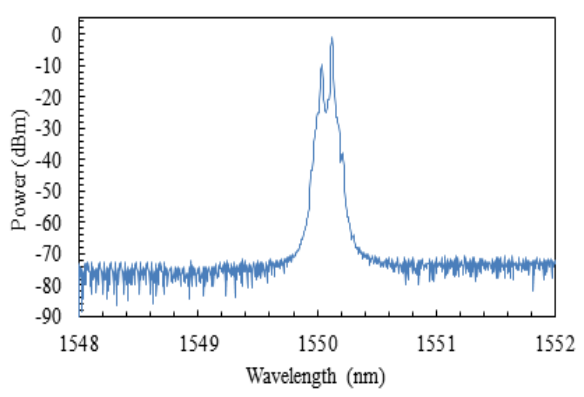

(a)

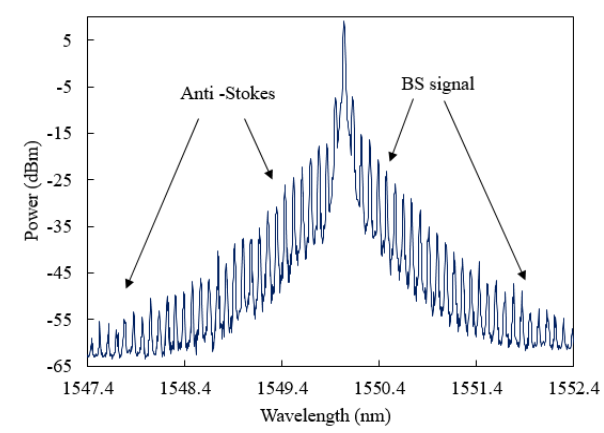

(c)

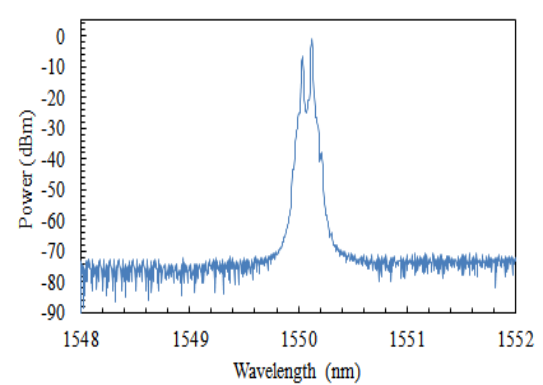

(b)

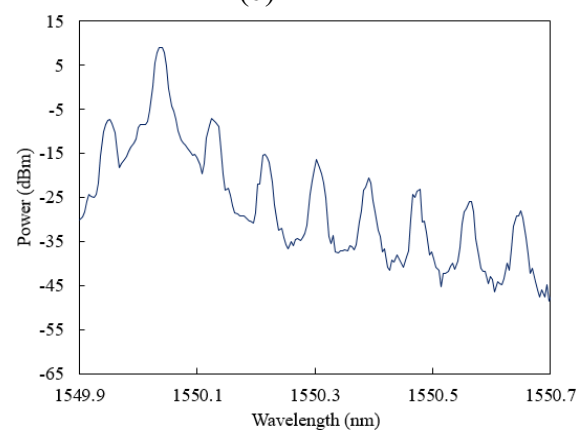

(d)

Fig. 3. The output spectra for different FBG locations in the BFL configuration. (a) The FBG is placed in the loop mirror after SMF, (b) the position of FBG in the loop mirror before SMF, (c) The position of the FBG at the end of the fiber configuration and (d) The BS signals with wavelength downshifted $\sim 0.08 \mathrm{~nm}$. 
As a result, the implementations of three different locations of FBG have yielded different output spectra as shown in Fig. 3. Based on the Fig. 3(a) and Fig. 3(b), when FBG is placed in the loop mirror neither before nor after the SMF, the single wavelength is produced even though the input power has been optimized and the threshold power has been achieved. Besides that, the position of the FBG at the end of the fiber laser has successfully demonstrated the multi-wavelength generations where the signals are reflected back by the FBG into the loop mirror as depicted Fig. 3(c).

This has successfully produced the generations of the BS signals with wavelength downshifted of $\sim 0.08 \mathrm{~nm}$ as shown in Fig. 3 (d). These spectra are generated at $1550 \mathrm{~nm}$ of BP wavelength with amplified BP power at $19.0 \mathrm{dBm}$. Characterization of the setup in Fig. 2 (c) where the FBG is placed at the end of the fiber laser is successfully obtained in this research. In order to investigate the number of BS signals, the $1550 \mathrm{~nm}$ of BP wavelength and amplified BP power of $16.0 \mathrm{dBm}$ to $20.0 \mathrm{dBm}$ are injected to the BFL configuration. From Fig. 4, $19.0 \mathrm{dBm}$ BP power and 50\% output ratio have been identified as the optimum parameters to produce a highest number of BS signals when compared with the other output coupling ratio. At these values, $23 \mathrm{BS}$ signal are produced. This condition can be explained that when the BP power increases the number of BS signals rises up by owing to sufficient energy is provided to generate higher order BS signals generation [19]. When the $\mathrm{BP}$ power increases at $20.0 \mathrm{dBm}$, the number of BS signals shows slight declination to 22. It is observed at $16.0 \mathrm{dBm}, 17.0 \mathrm{dBm}$ and $18.0 \mathrm{dBm}$ of BP powers, 15,14 and $20 \mathrm{BS}$ signals are generated, respectively. Meanwhile, when the values of output coupling ratio increase beyond $50 \%$, which is at $70 \%$ and $80 \%$, the number of BS signals is reduced. This trend is attributed from the increment of the cavity loss [20]. This factor affected the performance to generate higher number BS signals. At the lowest output coupling ratio of $20 \%, 1,5,6,9$ and $11 \mathrm{BS}$ signals are generated at $16.0 \mathrm{dBm}, 17 \mathrm{dBm}, 18.0 \mathrm{dBm}, 19.0 \mathrm{dBm}$ and $20.0 \mathrm{dBm}$, respectively. The output spectra at different coupling $(20 \%, 30 \%, 50 \%, 70 \%$ and $80 \%$ ) when $1550 \mathrm{~nm}$ of BP wavelength and $19.0 \mathrm{dBm}$ of BP power are injected inside the configuration is shown in Fig. 5.

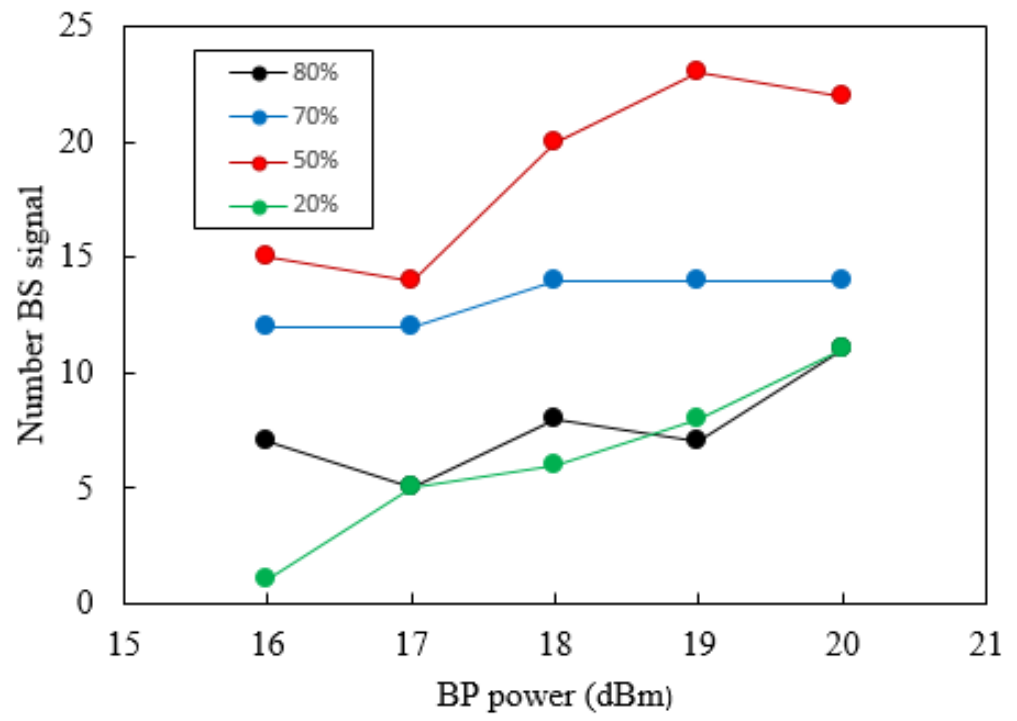

Fig. 4. Number of BS signal versus BP power at BP wavelength of $1550 \mathrm{~nm}$ with output coupling ratio of $80 \%, 70 \%, 50 \%$ and $20 \%$. 


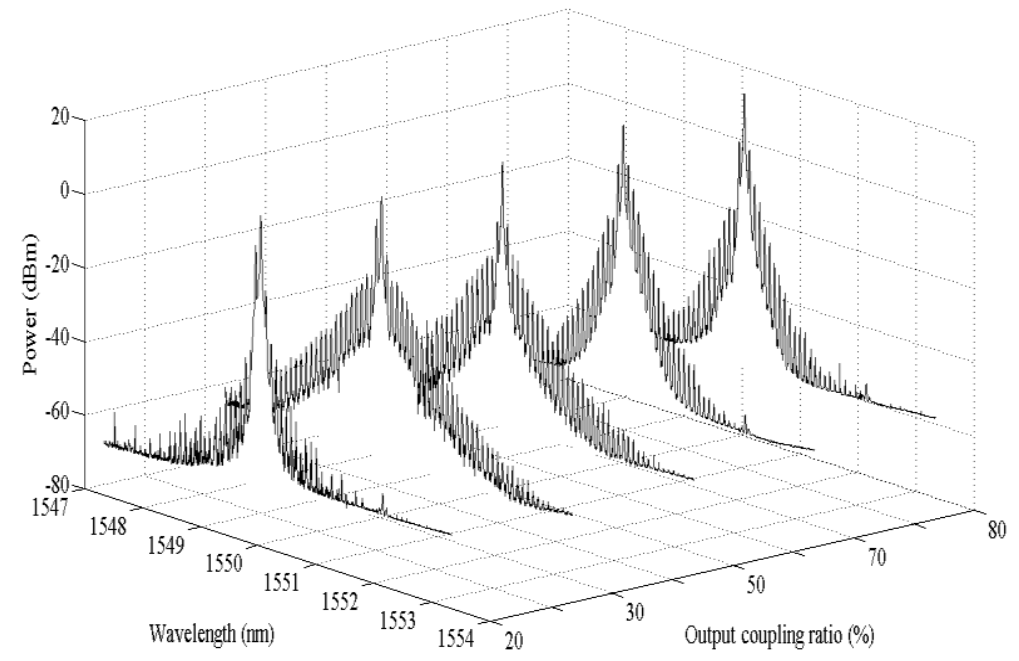

Fig. 5. The MWBFL spectra at different output coupling $(20 \%, 30 \%, 50 \%, 70 \%$ and $80 \%)$ when 1550 $\mathrm{nm}$ of BP wavelength and $20.0 \mathrm{dBm}$ of BP power are injected.

In order to observe the relationship between tuning range and multi-wavelength generation, produced optical spectra from $1548 \mathrm{~nm}$ to $1552 \mathrm{~nm}$ of BP wavelengths is plotted in Fig. 6. The amplified BP power is fixed at $20.0 \mathrm{dBm}$. Meanwhile, the optical spectra are extracted at $50 \%$ output coupling ratio. The MWBFL configuration has the ability to tune only $4 \mathrm{~nm}$ which is from $1548 \mathrm{~nm}$ to $1552 \mathrm{~nm}$ of BP wavelengths. At 1550 $\mathrm{nm}$ of BP wavelengths, $22 \mathrm{BS}$ signals are produced. As $1550 \mathrm{~nm}$ is the center wavelength of the FBG properties, it is attributed to the creation of higher number of BS signals. Meanwhile, 16, 18, 19, 18 BS signals are generated at $1548 \mathrm{~nm}, 1547 \mathrm{~nm}, 1551 \mathrm{~nm}$ and $1552 \mathrm{~nm}$, respectively.

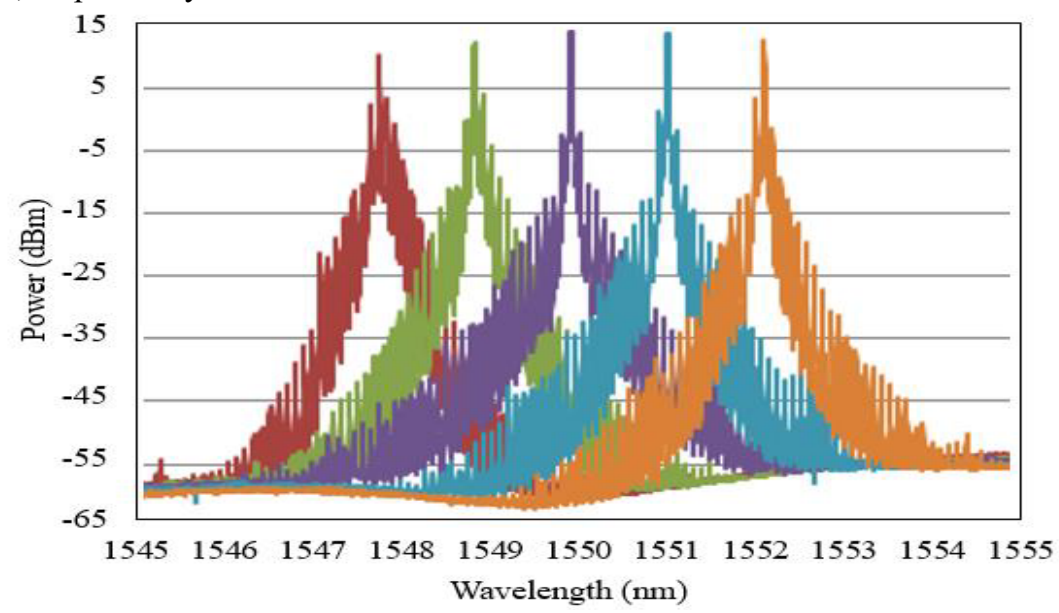

Fig. 6. Tuning ability of the MWBFL $1548 \mathrm{~nm}$ to $1552 \mathrm{~nm}$ of BP wavelength by injected $20.0 \mathrm{dBm}$ of amplified BP power at output coupling ratio of $50 \%$. 


\section{Summary}

As presented in this paper, the development of the MWBFL with FBG utilizing loop mirror is successfully demonstrated in the C-band region of $1530 \mathrm{~nm}$ to $1565 \mathrm{~nm}$. The position of the FBG in the MWBFL configuration plays the important role in producing multiwavelength signals. In addition, with injected BP power of $19.0 \mathrm{dBm}, 23 \mathrm{BS}$ signals and 4 $\mathrm{nm}$ of tuning range are obtained. Besides, the $50 \%$ output coupling ratio has the ability to generate higher number of BS signals. It can be concluded that the performance of the MWBFL utilizing loop mirror is dependent on the FBG location to produce multiwavelength signals.

This work was fully supported by the Ministry of Higher Education, Malaysia under research grant \# FRGS/9003-00532\#.

\section{References}

1. N.A.M Ahmad Hambali, N. Roshidah, M. SafuanZainudin, A. ZakiahMalek, M.H.A. Wahid, M. Shakirin Abu Hurera, International Conference on Electronic Design (ICED), Penang, Malaysia (2014)

2. M. Shakirin Abu Hurera, Development of L-band ring cavity Brillouin Fiber Laser, Universiti Malaysia Perlis, Perlis, Malaysia (2015)

3. R.J. Ducar, Particle Accelerator Conference, Accelerator Science and Technology, San Francisco, CA, US (1991)

4. G. Snell, SMPTE Journal, 105(1), 4 (1996)

5. K.Kikuchi, Journal of Lightwave Technology, 34, 157 (2016)

6. M.J. Hackerr, O. Muja, Avionics Fiber-Optics and Photonics IEEE Conference, Annapolis, US (2006)

7. S.W. Harun, IEEE Journal in Quantum Electronics, 20, 1100108 (2014)

8. D. Schuocker, G. Liedl, A. Kratky, University of Technology, Vienna, Austria (2013)

9. D.Choudhury, A.K. Kar, R. Mary, IEEE Journal of Selected in Quantum Electronics, 20 (2014)

10. M.E. Fermann, I. Hartl, Nature Photonics, 7, 868 (2013)

11. M. Giesberts, S. Nyga, O. Fitzau, B. Jungbluth, H.D. Hoffmann, O. Bang C. Larsen, Optics Express, 21, 12303 (2013)

12. D.W. Hahn, N. Omenetto, SAGE Journal, 66, 347 (2012)

13. H. Gong, L. Zhang, Y. Jin, J. Wang, Y. Tu, International Conference on Electronics and Optoelectronics (2011)

14. Z. Yusoff, M.H. Al-Mansoori, H.A. Abdul Rashid, P.K. Choudhury, M.N. Mohd Nasir, National Conference on Telecommunication Technologies and IEEE 2008 2nd Malaysia Conference on Photonics (2008)

15. N.H. Muhammad Apandi, MultiwavelengthBrillouin Fiber Laser with Fiber Bragg Grating utilizing loop mirror, Universiti Malaysia Perlis, Perlis, Malaysia (2015)

16. A. Buxens, A.T. Clausen, P. Jeppesen, H.N. Poulsen, Lasers and Electro-Optics, 39, 277 (2000)

17. R.J. Mears, Fifth Asia-Pacific Conference, Beijing, China (1999)

18. Y. Makiuchi, H. Matsuura, Development of Low Loss Optical Circulator, Furukawa Electric Review, 59, 1-4 (2002) 
19. N.A.M Ahmad Hambali, M. Ajiya, M.A Mahdi, R. Sonee Shargh, Journal of Optics, 17 (2015)

20. M.A. Mahdi, M.H. Al-Mansoori, M.I. Saripan, A.F. Abas, N.A.M Ahmad Hambali, Applied Optics, 48, 5055 (2009) 